\title{
Change Point Analysis and Clustering Examined Through Chicago Crime During COIVD-19
}

\author{
Mena Whalen ${ }^{1}$, Dr. Andrew Papachristos ${ }^{2}$, Dr. Joseph Feinglass ${ }^{3}$, Dr. Noelle I. Samia ${ }^{1}$ \\ ${ }^{1}$ Northwestern University Department of Statistics \\ 2006 Sheridan Rd. Evanston IL United States \\ mcwhalen@u.northwestern.edu; n-samia@northwestern.edu \\ ${ }^{2}$ Northwestern University Department of Sociology \\ 1810 Chicago Avenue, Evanston, IL United States \\ avp@northwestern.edu \\ ${ }^{3}$ Northwestern University Department of Preventive Medicine \\ 750 N Lake Shore, Chicago Illinois United States \\ j-feinglass@northwestern.edu
}

\section{Extended Abstract}

The COVID-19 pandemic has created shifts to daily life and changed human interactions across the globe. Possibly leading to a shift in a time series but given the ever-evolving nature of the pandemic; where is the shift, are there multiple changes, and how these shifts change across locations? Using change point analysis allows for the data to determine where a change in mean, or other parameters, occurred. We develop spatio-temporal change point methodologies to investigate when Index crime rates changed in Chicago, IL, using weekly time series from 77 community areas. Locations with similar temporal behaviour and spatial demographics are clustered together using a modified clustering algorithm that enables clustering based on similar change point locations and spatial characteristics. Through specialized diagnostic measures and inventive data visualizations each unique aspect of the data is analysed. 